\title{
Novel Band-Reject FiLTER Design USING MULTILAYER BRAGG MIRROR AT 1550 NM
}

\author{
Krishanu Nandy ${ }^{1}$, Suhrid Biswas ${ }^{2}$, Rahul Bhattacharyya ${ }^{3}$, \\ Soumendra Nath Saha ${ }^{4}$, Arpan Deyasi ${ }^{5}$ \\ 1,2,3,4,5 Department of Electronics of Communication Engineering, RCC Institute \\ of Information Technology, Canal South Road, Kolkata-700015 \\ \{krishrcc91, suhridbiswas3, rahulrcciit91\} egmail.com \\ ${ }^{4}$ soumendra91@yahoo.com, ${ }^{5}$ deyasi_arpan@yahoo.co.in
}

\begin{abstract}
Novel band-reject filter is proposed using multilayer Bragg mirror structure by computing reflection coefficient at $1550 \mathrm{~nm}$ wavelength for optical communication. Dimension of different layers and material composition are modified to study the effect on rejection bandwidth, and no. of layers is also varied for analyzing passband characteristics. $G a N / A l_{x} G a_{1-x} N$ composiiton is taken as the choice for simulation purpose, carried out using propagation matrix method. Refractive indices of the materials are considered as function of bandgap, operating wavelength and material composition following Adachi's model. One interesting result arises from the computation that band-reject filter may be converted into band-pass one by suitably varying ratio of thicknesses of unit cell, or by varying Al mole fraction. Simulated results can be utilised to design VCSEL mirror as optical transmitter.
\end{abstract}

\section{KEYWORDS}

Bragg mirror, Reflection coefficient, Multilayer structure, Band-reject filter

\section{INTRODUCTION}

Bragg reflectors have already attracted interest of researchers for their possible novel applications as optical transmitter [1-2], LED [3], reflectivity modulators [4], solar cell [5]. Highly transparent dielectric materials are required are used to construct Bragg mirrors in order to minimize the loss due to optical absorption [6-8]. It is a structure formed from multiple layers of alternating materials with periodic variation of refractive index, where each layer boundary causes a partial reflection of an optical wave. For waves whose wavelength is approximately four times the optical thickness of the layers, reflections then combine with constructive interference, and the layers act as a high-quality reflector. For design of such highly reflecting multilayer structure using dielectric materials, its reflectivity plays a critical role along with dispersion and phase [9]. By suitably choosing the refractive indices of the constituting layers along with dimension of slabs, required filter characteristics can be obtained from reflection coefficient profile; when computed for the desired frequency region. Depending on the position of passband, it can be utilized as band-reject or bandpass filter, depending on the requirement for optical communication purpose.

In this paper, reflectivity of multilayer Bragg mirror is computed using propagation matrix method at $1550 \mathrm{~nm}$ considering $\mathrm{GaN} / \mathrm{Al}_{\mathrm{x}} \mathrm{Ga}_{1-\mathrm{x}} \mathrm{N}$ material composiiton. It is observed that by suitably choosign dimension of slabs and $\mathrm{Al}$ mole fraction of $\mathrm{Al}_{\mathrm{x}} \mathrm{Ga}_{1-\mathrm{x}} \mathrm{N}$ layer, the structure can be

Rupak Bhattacharyya et al. (Eds) : ACER 2013,

pp. 419-425, 2013. (C) CS \& IT-CSCP 2013

DOI : $10.5121 /$ csit.2013.3239 
used as band-reject or bandpass filter. Rsults can be utilized to design multilayer VCSEL structure.

\section{Mathematical Modelling}

We consider the simplest three layers having refractive indices as shown in Fig 1:

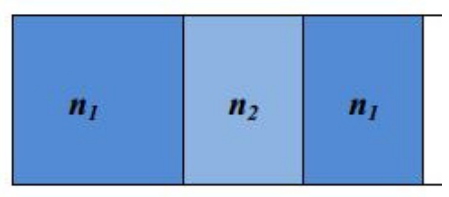

Figure 1: Three-layer interface with $\mathrm{r}$. $\mathrm{i}$ as $\mathrm{n}_{1}, \mathrm{n}_{2}, \mathrm{n}_{1}$

We consider wavevectors $\mathrm{k}_{0}, \mathrm{k}_{1}, \mathrm{k}_{2}$ defined as in terms of wavelength, and assume normal incidence of light on the structure. For wave at media interface having propagation constant $k_{1}$ and $\mathrm{k}_{2}$ respectively, satisfaction of boundary conditions give the following equation-

$$
A_{1} \exp \left(-i k_{1} z\right)+A_{2} \exp \left(i_{1} z\right)=B_{1} \exp \left(-i_{2} z\right)+B_{2} \exp \left(i_{2} z\right)
$$

Then interface matrices can in general be represented as-

$$
D_{i}=\left[\begin{array}{cc}
1 & 1 \\
k_{i} & -k_{i}
\end{array}\right]
$$

where

$$
k_{i}=\frac{2 \pi}{\lambda} n_{i}
$$

Considering propagation matrices as $\mathrm{P}_{1}, \mathrm{P}_{2}, \mathrm{P}_{3}$; we can write it in general-

$$
P_{i}=\left[\begin{array}{cc}
\exp \left(-j k_{i} d_{i}\right) & 0 \\
0 & \exp \left(j k_{i} d_{i}\right)
\end{array}\right]
$$

Gain can be calculated as-

$$
G_{i}=\left(D_{i} P_{i}\left(D_{i}\right)^{-1}\right)\left(D_{i+1} P_{i+1}\left(D_{i+1}\right)^{-1}\right)
$$

\section{Material Parameter}

Several models already have been reported to calculate refractive index but up to now Adachi's model is considered most accurate model. Several research groups have proposed own values of Adachi's fitting parameters that give different level of accuracy for a range of composition of $\mathrm{Al}$ in nitride alloys. The continuous equation to calculate the refractive index for all nitrides material proposed by Adachi and later simplified [10-11] is given below: 


$$
n(E)=\sqrt{\left\{A\left(\frac{\hbar \omega}{E_{g}}\right)^{-2}\left\{2-\sqrt{\left(1+\left(\frac{\hbar \omega}{E_{g}}\right)\right)}-\sqrt{\left(1-\left(\frac{\hbar \omega}{E_{g}}\right)\right)}\right\}+B\right\}}
$$

where $E_{g}$ is the bandgap energy of the material, $\omega$ is the frequency of the laser emission and $A$ and $\mathrm{B}$ are the fitting parameters. For simulation purpose, we have considered $\mathrm{GaN} / \mathrm{Al}_{\mathrm{x}} \mathrm{Ga}_{1-\mathrm{x}} \mathrm{N}$ composition.

\section{RESUlTS \& DisCUSSION}

Using the equations, reflectance of Bragg's mirror is computed for multilayer structure by varying thicknesses and material composition of the layers, as well as no of layers also. Fig 2a shows the reflection coefficient of 11-layer structure centered at $1.55 \mu \mathrm{m}$, and by suitably choosing the ratio of thickness of the unit cell and also material composition, reflectivity can be made unity near the desired frequency range of interest. Hence it can be considered as a band-reject filter for noise. It is also observed that by increasing no. of layers, bandwidth slightly increases; and reflectivity at passbands monotonically decreases following exponential nature. This is plotted in Fig $2 \mathrm{~b}$.

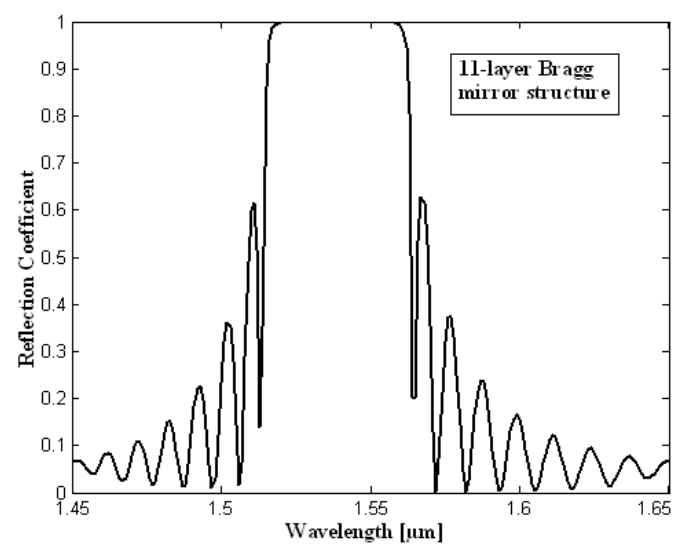

Figure 2a: Reflection coefficient with wavelength centered at $1.55 \mu \mathrm{m}$ for 11-layer Bragg mirror structure

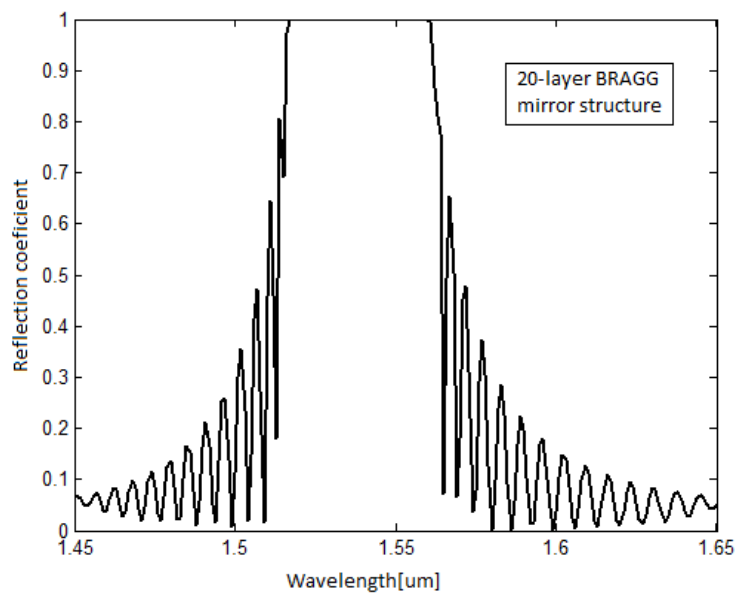

Figure 2b: Reflection coefficient with wavelength centered at $1.55 \mu \mathrm{m}$ for 20-layer Bragg mirror structure 
If material composition is changed for that multilayer structure keeping no. of layers constant, then it is observed that with increases of $\mathrm{Al}$ mole fraction, rejection bandwidth at the desired central wavelength increases, i.e., filter becomes wideband one. This is shown in Fig 3b \& Fig 3c respectively. Interestingly, it can also be observed that reduction of $\mathrm{Al}$ percentage changes the nature of the filter, and it becomes bandpass one. This observation is plotted in Fig 3a.

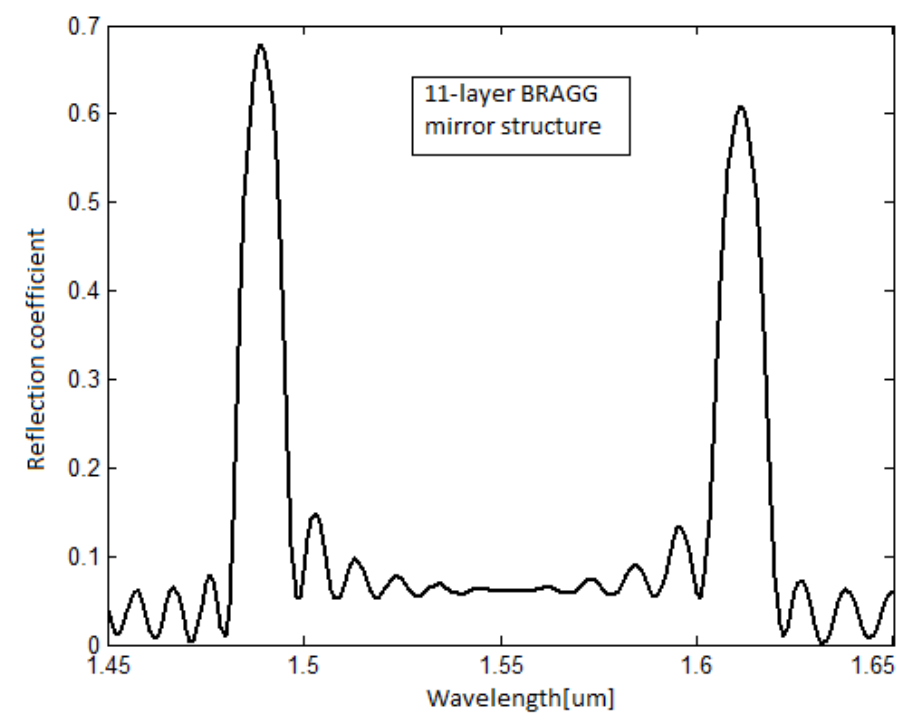

Figure 3a: Reflection coefficient with wavelength centered at $1.55 \mu \mathrm{m}$ for 11-layer Bragg mirror structure with $\mathrm{Al}_{0.4} \mathrm{Ga}_{0.6} \mathrm{~N} / \mathrm{GaN}$ composition

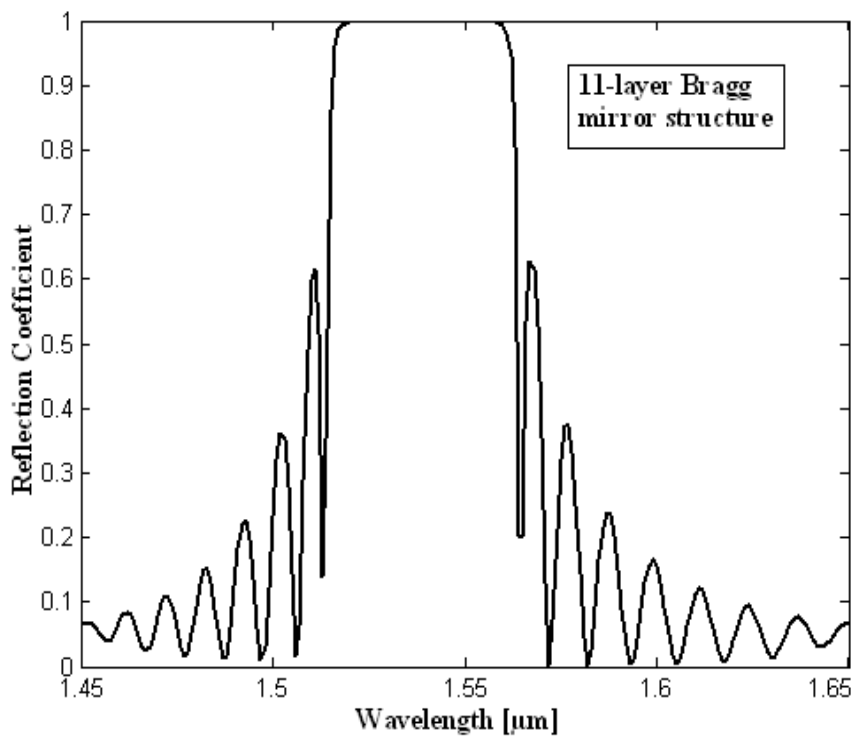

Figure 3b: Reflection coefficient with wavelength centered at $1.55 \mu \mathrm{m}$ for 11-layer Bragg mirror structure with $\mathrm{Al}_{0.6} \mathrm{Ga}_{0.4} \mathrm{~N} / \mathrm{GaN}$ composition 


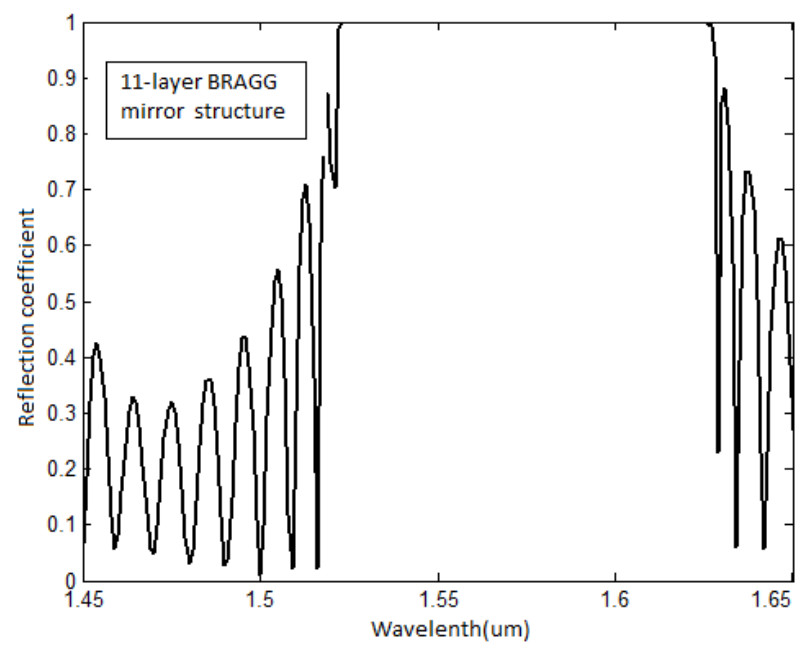

Figure 3c: Reflection coefficient with wavelength centered at $1.55 \mu \mathrm{m}$ for 11-layer Bragg mirror structure with $\mathrm{Al}_{0.7} \mathrm{Ga}_{0.3} \mathrm{~N} / \mathrm{GaN}$ composition

By varying the dimension of different layers keeping no. of layers and material composition constant, it is observed that increasing the width of either of the layer ultimately shift the predetermined filter bandwidth from the desired optical communication wavelength and hence optimization of layer thickness is very much essential. In Fig $4 \mathrm{a}$, reflection coefficient is plotted with variation of $\mathrm{Al}_{x} \mathrm{Ga}_{1-\mathrm{x}} \mathrm{N}$ layer thickness. It is observed that the filter characterisitcs for 11layer structure with $\mathrm{Al}_{0.6} \mathrm{Ga}_{0.4} \mathrm{~N} / \mathrm{GaN}$ composition is optimized for $3.5 \mu \mathrm{m}$ width, and decreasing or increasing the dimension converts it in a bandpass filter. This effect is simulated considering the $\mathrm{GaN}$ thickness as $2.7 \mu \mathrm{m}$. Now keeping thickness of $\mathrm{Al}_{0.6} \mathrm{Ga}_{0.4} \mathrm{~N}$ layer as $3.5 \mu \mathrm{m}$, if width of $\mathrm{GaN}$ layer is varied, then also filter characteristic changes; as evident from Fig $4 \mathrm{~b}$.

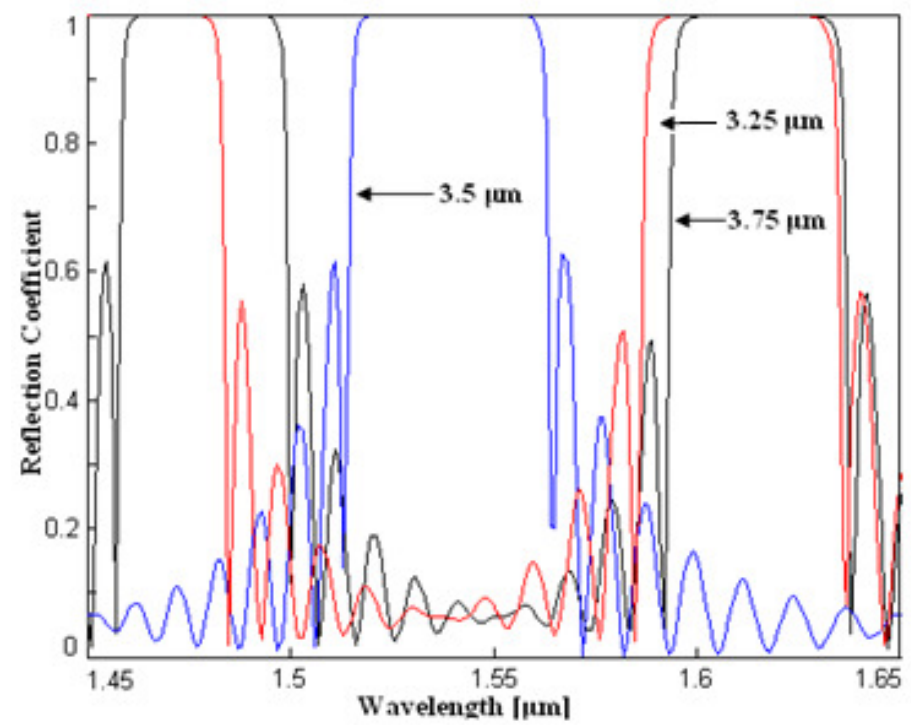

Figure 4a: Reflection coefficient with wavelength centered at $1.55 \mu \mathrm{m}$ for 11-layer Bragg mirror with $\mathrm{Al}_{0.6} \mathrm{Ga}_{0.4} \mathrm{~N} / \mathrm{GaN}$ composition for different thickness of $\mathrm{Al}_{0.6} \mathrm{Ga}_{0.4} \mathrm{~N}$ but constant $\mathrm{GaN}$ thickness 


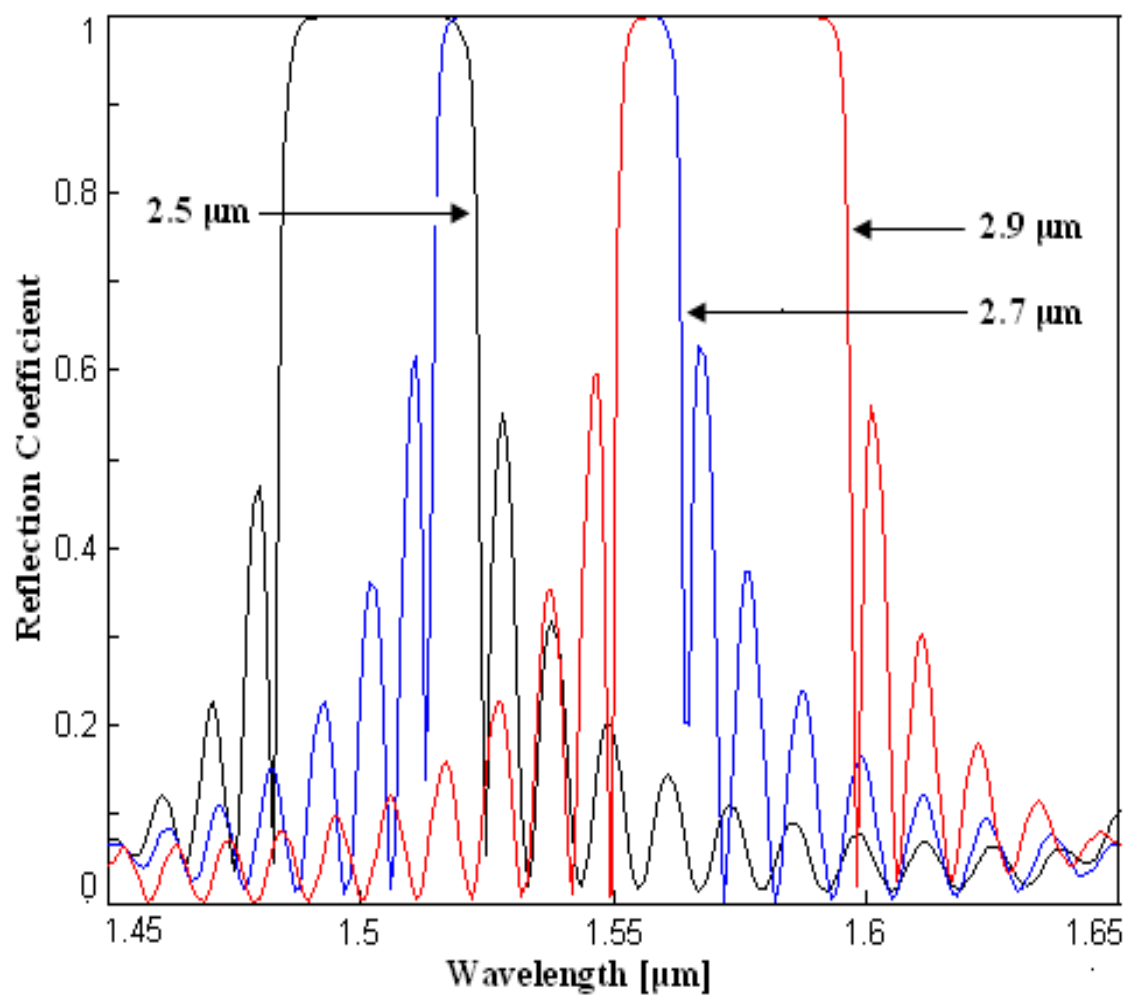

Figure 4b: Reflection coefficient with wavelength centered at $1.55 \mu \mathrm{m}$ for 11-layer Bragg mirror with $\mathrm{Al}_{0.6} \mathrm{Ga}_{0.4} \mathrm{~N} / \mathrm{GaN}$ composition for different $\mathrm{GaN}$ thickness of but constant $\mathrm{Al}_{0.6} \mathrm{Ga}_{0.4} \mathrm{~N}$ thickness

\section{CONCLUSION}

Throughout the simulation work, filter property of multilayer Bragg mirror structure is analyzed by means of reflection coefficient at $1550 \mathrm{~nm}$. Dimension of different layers and material compositions are varied to observe the effect on optical property. Numbers of layers are varied to observe the variation of width of desired region. In this work we considered the dependence of refractive indices of different layers on material composition and frequency of interest. Propagation matrix method is considered for material analysis. Optimized profile is generated for the structure combining the effect of number of layers, their dimension and material composition. Results are very helpful to analyze vertical cavity surface emitting laser analysis.

\section{REFERENCES}

[1] M. Achtenhagen, N. V. Amarasinghe, L. Jiang, J. Threadgill, P. Youn, (2009), "Spectral Properties of High-Power Distributed Bragg Reflector Lasers", Journal of Lightwave Technology, vol. 27, pp. 3433-3437.

[2] M. Achtenhagen, N.V. Amarasinghe, G.A. Evans, (2007), High-power Distributed Bragg Reflector Lasers Operating at 1065nm", Electronic Letters, vol. 43.

[3] D. Wang, I. T. Ferguson, J. A. Buck, (2007), GaN-based Distributed Bragg Reflector for HighBrightness LED and Solid-State Lighting", Applied Optics, vol. 46, pp. 4763-4767.

[4] J. Y. Tsai, T. C. Lu, S. C. Wang, (2003), "High Reflectivity Distributed Bragg Reflectors for $1.55 \mu \mathrm{m}$ VCSELs using InP/Airgap", Solid State Electronics, vol. 47, pp. 1825-1828. 
[5] Y. Shimizu, Y. Okada, (2003), "GaAs/Si Solar Cells with Internal Bragg Reflector Superlattice Structure", Proceedings of 3rd World Conference on Photovoltaic Energy Conversion, vol. 1, 781784.

[6] A. V. Kavokin, M. A. Kaliteevski, (1996), "Light absorption Effect on bragg Interference in Multilayer Semiconductor Heterostructures", Journal of Applied Physics, vol. 79, pp. 595-598.

[7] V. V. Evstropov, M. A. Kaliteevski, A. L. Lipko, M. A. Sinitsyn, B. V. Tsarenkov, Y. M. Shernyakov, B. S. Yavich, (1996), "Semiconductor bragg reflector with absorbing Layers", Semiconductors, Vol 30, pp.57-59.

[8] J. L. Shen, C. Y. Chang, H. C. Liu, W. C. Chou, Y. F. Chen, T. Jung, M. C. Wu, (2001), "Reflectivity and Photoluminescence Studies in Bragg Reflectors with Absorbing Layers", Semiconductor Science and Technology, vol 16, pp. 548-552.

[9] J. L. Chen, C. Y. Chang, W. C. Chou, (2001), "Temperature Dependence of the Reflectivity in Absorbing Bragg Reflectors", Optics express, vol. 9, pp. 287-292.

[10] M. Gudan, J. Piprek, (1996), "Material Parameters of Quaternary III-V Semiconductors for Multilayer mirrors at 1.55 Micron wavelength", Modelling and Simulation of Material Science Engineering, vol. 4, pp. 349-357.

[11] G. M. Laws, E. C. Larkins, I. Harrison, C. Molloy, D. Somerford, (2001), "Improved Refractive index Formulas for the AlGaN and InGaN Alloys", Journal of applied Physics, vol. 89, pp. 1108-1115. 